\title{
EFEITOS DO EXTRATO DE Eugenia uniflora SOBRE PARÂMETROS METABÓLICOS E PERFIL LIPIDICO DE RATOS HIPERTENSOS
}

\author{
Noemia Aparecida Partelli Mariani ${ }^{1}$ \\ Karen Silva Rangel ${ }^{2}$ \\ Emily Neves Souza ${ }^{3}$ \\ David Chaves Felicio da Silva ${ }^{4}$ \\ Eduardo Frizzera Meira ${ }^{5}$ \\ Alessandra Simão Padilha ${ }^{6}$ \\ Juliana Aparecida Severi ${ }^{7}$ \\ Fabiana Dayse Magalhães Siman ${ }^{8}$
}

\begin{abstract}
Resumo: A Eugenia uniflora é uma planta amplamente distribuída no Brasil, utilizada popularmente para diversas desordens. O objetivo do trabalho foi avaliar os efeitos do extrato de suas folhas sobre o perfil lipídico e parâmetros metabólicos de Ratos Espontaneamente Hipertensos (SHR). Os animais foram divididos em grupos controle e tratados com extrato nas doses de $100 \mathrm{mg} / \mathrm{kg}$ e 200 $\mathrm{mg} / \mathrm{kg}$. O tratamento foi diário, via gavagem, por 28 dias. A pesagem dos animais foi semanal, e ao final, os animais foram colocados em gaiolas metabólicas por 24 horas para a quantificação de urina, ingesta de água e ração. Após jejum de 12 horas, foi realizada coleta de sangue para a determinação de HDL, LDL, Colesterol total e triglicerídeos. O extrato não modificou o consumo de água e ração, eliminação de urina e peso corporal. Porém, diminuiu a concentração de LDL e triglicerídeos no grupo de $200 \mathrm{mg} / \mathrm{Kg}$ e $100 \mathrm{mg} / \mathrm{Kg}$.
\end{abstract}

Palavras-chave: Eugenia uniflora; Perfil lipídico; Parâmetros metabólicos; SHR.

\footnotetext{
${ }^{1}$ Farmácia/Universidade Federal do Espírito Santo - Centro de Ciências Exatas Naturais e da Saúde, Brasil. Email: noemiapartelli@hotmail.com.

${ }^{2}$ Farmácia/Universidade Federal do Espírito Santo - Centro de Ciências Exatas Naturais e da Saúde, Brasil. Email: karenrangelfarm@gmail.com.

3 Farmácia/Universidade Federal do Espírito Santo - Programa de Pós-Graduação em Ciências Fisiológicas, Universidade Federal do Espírito Santo, Brasil. E-mail: neves.emilly@hotmail.com.

${ }^{4}$ Farmácia/Universidade Federal do Espírito Santo - Programa de Pós-Graduação em Ciências Fisiológicas, Universidade Federal do Espírito Santo, Brasil. E-mail: felicio762@gmail.com.

${ }^{5}$ Farmácia/Universidade Federal do Espírito Santo - Programa de Pós-Graduação em Ciências Fisiológicas, Universidade Federal do Espírito Santo, Brasil. E-mail: eduardo.frizzera.meira@gmail.com.

6 Farmácia/Universidade Federal do Espírito Santo - Programa de Pós-Graduação em Ciências Fisiológicas, Universidade Federal do Espírito Santo, Brasil. E-mail: ale_padilha@hotmail.com.

${ }^{7}$ Farmácia/Universidade Federal do Espírito Santo - Centro de Ciências Exatas Naturais e da Saúde, Brasil. Email: juseveri@yahoo.com.br.

${ }^{8}$ Farmácia/Universidade Federal do Espírito Santo - Centro de Ciências Exatas Naturais e da Saúde, Brasil. Email: famisiman@gmail.com.
} 